\title{
ON HOMOGENEOUS POLYNOMIALS ON A COMPLEX BALL
}

BY

\author{
J. RYLL AND P. WOJTASZCZYK
}

\begin{abstract}
We prove that there exist $n$-homogeneous polynomials $p_{n}$ on a complex $d$-dimensional ball such that $\left\|p_{n}\right\|_{\infty}=1$ and $\left\|p_{n}\right\|_{2} \geqslant \sqrt{\pi} 2^{-d}$. This enables us to answer some questions about $H_{p}$ and Bloch spaces on a complex ball. We also investigate interpolation by $n$-homogeneous polynomials on a 2 -dimensional complex ball.
\end{abstract}

Introduction. The starting point of our investigation was a question asked by $\mathrm{S}$. Waigner: Is the identity map from $H_{\infty}\left(B_{d}\right)$ into $H_{1}\left(B_{d}\right)\left(B_{d}\right.$ is a unit ball in $\left.C^{d}\right)$, $d>1$, a compact linear map. This question has a connection with the well-known open problem (cf. [5]): does there exist a nonconstant inner function on $B_{d}, d>1$ ? $^{1}$ The existence of such an inner function would imply our Corollary 1.5, namely that this operator is not compact. We obtain this result by exhibiting $n$-homogeneous polynomials $p_{n}$ which in some respects resemble the monomials $z^{n}$ in the one-dimensional case (Theorem 1.2). We give two proofs of this theorem, one in $\S 1$ and the other at the end of $\S 2$. Those polynomials enable us to also answer a question of $\mathbf{R}$. Timoney (Corollary 1.9). We hope that they will find some other applications. In $\$ 2$ we investigate interpolating $n$-homogeneous polynomials on the unit sphere in two-dimensional complex space. The motivation for this study is the following well-known open problem (cf. [6]): does there exist a function $\varphi(t), t \geqslant 1$, such that for every finite-dimensional Banach space $X$ we have $d\left(X, l_{\infty}^{\operatorname{dim} X}\right) \leqslant \varphi(\lambda(X)$ ) (for the definitions, see below). To the best of our knowledge, the spaces $W_{n}^{\infty}\left(S_{2}\right)$, of all $n$-homogeneous polynomials on the unit ball in $\mathbf{C}^{2}$, are the first spaces known for which $\lambda\left(W_{n}^{\infty}\left(S_{2}\right)\right)$ is bounded independently of $n$, while $d\left(W_{n}^{\infty}\left(S_{2}\right), l_{\infty}^{n+1}\right)$ is not known to be bounded. Thus the following problem naturally arises.

Problem. Compute or estimate $d\left(W_{n}^{\infty}\left(S_{d}\right), l_{\infty}^{k}\right), k=\operatorname{dim} W_{n}^{\infty}\left(S_{d}\right)$.

In our opinion, the results of $\$ 2$ indicate that this problem may not be trivial. ${ }^{2}$

Apart from this Banach space motivation our results may be interesting as interpolation results. They are very different from corresponding results for trigonometric polynomials on the unit circle, of degree at most $n$ (cf. [8, Chapter X]).

Now let us fix some notations. We will work with $\mathbf{C}^{d}$, the complex $d$-dimensional space equipped with the usual scalar product denoted by $\langle\cdot, \cdot\rangle . B_{d}$ will stand for the

\footnotetext{
Received by the editors May 4, 1981.

1980 Mathematics Subject Classification. Primary 32A05, 41A05; Secondary 32A35, 46B20.

'After this paper was accepted A. B. Alexandrov has constructed a nontrivial inner function on the ball, see The existence of inner functions on the ball, Mat. Sbornik 118 (160) (1982), 147-163. (Russian)

${ }^{2}$ The approach indicated in the last remark of this paper was illuminating to $\mathrm{R}$. Bourgain who used it to show that $d\left(W^{n}\left(S_{2}\right), l_{\infty}^{n+1}\right) \leqslant c(\log n)^{2}$.
} 
unit ball in $\mathbf{C}^{d}$, i.e. $B_{d}=\left\{\zeta \in \mathbf{C}^{d}:\langle\zeta, \zeta\rangle \leqslant 1\right\}$ and $S_{d}$ will stand for the unit sphere in $\mathbf{C}^{d}$, i.e. $S_{d}=\left\{\zeta \in \mathbf{C}^{d}:\langle\zeta, \zeta\rangle=1\right\}$. The point $(1,0, \ldots, 0) \in S_{d}$ will be denoted by 1 . On $S_{d}$ we have the natural rotation-invariant probability measure $\sigma . W_{n}\left(S_{d}\right)$ will denote the space of all $n$-homogeneous polynomials on $\mathrm{C}^{d}$ restricted to $S_{d}$. On $W_{n}\left(S_{d}\right)$ we will consider various norms. For $f \in W_{n}\left(S_{d}\right)$ we put $\|f\|_{p}=$ $\left(\int_{S_{d}}|f(\zeta)|^{p} d \sigma(\zeta)\right)^{1 / p}$ if $1 \leqslant p<\infty$ and $\|f\|_{\infty}=\sup _{\zeta \in S_{d}}|f(\zeta)|$. The Banach space $\left(W_{n}\left(S_{d}\right),\|\cdot\|_{p}\right)$ will be denoted by $W_{n}^{p}\left(S_{d}\right)$. The symbols $L_{p}\left(S_{d}\right)$ and $L_{\infty}\left(S_{d}\right)$ have natural meaning. The closure of all polynomials in $L_{p}\left(S_{d}\right)$ will be denoted by $H_{p}\left(B_{d}\right)$ and $H_{\infty}\left(B_{d}\right)$ will denote the space of all bounded analytic functions on $B_{d}$. Obviously for $n=0,1,2, \ldots, W_{n}^{p}\left(S_{d}\right)$ is naturally a subspace of $H_{p}\left(B_{d}\right)$. For information about $H_{p}\left(B_{d}\right)$ the reader may consult [5].

1. In this section we prove the main result of the paper and give some applications to the structure of $H_{p}$ spaces. We introduce the notation

$$
\alpha(d, p)=\int_{S_{d}}|\langle\zeta, 1\rangle|^{p} d \sigma(\zeta)
$$

It can be computed that $\alpha(d, p)=\Gamma((1+p) / 2) \Gamma(d) / \Gamma(d+p / 2)$.

Proposition 1.1. The operator

$$
\left(P_{n} f\right)(\zeta)=\alpha(d, 2 n)^{-1} \int_{S_{d}} f(\eta)\langle\zeta, \eta\rangle^{n} d \sigma(\eta)
$$

is an orthogonal projection from $L_{2}\left(S_{d}\right)$ onto $W_{n}^{2}\left(S_{d}\right)$. The norm of this projection considered as an operator from $L_{\infty}\left(S_{d}\right)$ onto $W_{n}^{\infty}\left(S_{d}\right)$ is smaller than $2^{d-1}$.

Proof. We will consider the natural representation of $U(d)$ (the group of all unitary operators on $\left.\mathbf{C}^{d}\right)$ on $L_{2}\left(S_{d}\right)$ defined by $g \mapsto T_{g}, T_{g} f(\zeta)=f\left(g^{-1} \zeta\right)$. The spaces $W_{n}^{2}\left(S_{d}\right)$ are invariant, irreducible spaces for this representation. Since $P_{n}$ commutes with this representation and $\operatorname{Im} P_{n} \subset W_{n}^{2}\left(S_{d}\right)$, we infer that $P_{n} \mid W_{n}^{2}\left(S_{d}\right)$ $=\lambda$ Id. If we put $f(\eta)=\langle\eta, 1\rangle^{n}$ we obtain $\lambda f(\mathbf{1})=\left(P_{n} f\right)(\mathbf{1})=f(\mathbf{1})$, so $\lambda=1$. This means that $P_{n}$ is an orthogonal projection onto $W_{n}^{2}\left(S_{d}\right)$. Its norm as an operator on $L_{\infty}\left(S_{d}\right)$ equals $\alpha(d, n) \alpha(d, 2 n)^{-1} \leqslant 2^{d-1}$.

THEOREM 1.2. For every $n$ there exists $p_{n} \in W_{n}\left(S_{d}\right)$ with $\left\|p_{n}\right\|_{\infty}=1$ and $\left\|p_{n}\right\|_{2}$ $\geqslant \sqrt{\pi} 2^{-d}$.

Before we start the proof of Theorem 1.2 let us recall some well-known notions concerning finite-dimensional Banach spaces. Let $X, Y$ be finite-dimensional Banach spaces. We put

$\lambda(X)=\inf \left\{\|T\| \cdot\|S\|: X \stackrel{T}{\rightarrow} L_{\infty} \stackrel{S}{\rightarrow} X, T S=\operatorname{Id}_{X}, L_{\infty}\right.$ is an arbitrary $L_{\infty}(\mu)$-space $\}$

and

$$
d(X, Y)=\inf \left\{\|T\| \cdot\left\|T^{-1}\right\|: T: X \rightarrow Y \text { is } 1-1 \text { and onto }\right\}
$$


The number $\lambda(X)$ is called a projection constant of $X$ and $d(X, Y)$ is called the Banach-Mazur distance between $X$ and $Y$. It is well known and easy to see that

$$
\lambda(X) \leqslant d(X, Y) \lambda(Y)
$$

Moreover we have

$$
\lambda\left(l_{2}^{n}\right)=\alpha(n, 1) \alpha(n, 2)^{-1} \geqslant \frac{1}{2} \sqrt{\pi} \sqrt{n} .
$$

More information on this can be found in [6] and [4, 22.1 and 28.1].

We will also use the following two lemmas.

LEMMA 1.3. $\inf \left\{\|p\|_{2}: p \in W_{n}\left(S_{d}\right)\right.$ and $\left.\|p\|_{\infty}=1\right\}=\sqrt{\alpha(d, 2 n)}$.

Proof. Since the spaces under consideration are finite dimensional, there exists a polynomial $p_{0}$ realising the minimum. We can assume $p_{0}(\mathbf{1})=1=\left\|p_{0}\right\|_{\infty}$. It is easily seen that

$$
p(\zeta)=\frac{1}{(2 \pi)^{d-1}} \int_{0}^{2 \pi} \cdots \int_{0}^{2 \pi} p_{0}\left(z_{1}, e^{i \theta_{1}} z_{2}, \ldots, e^{i \theta_{d-1}} z_{d}\right) d \theta_{1} \cdots d \theta_{d-1}=\langle\zeta, 1\rangle^{n} .
$$

Moreover, $\|p\|_{2} \leqslant\left\|p_{0}\right\|_{2}$ and $\|p\|_{\infty}=\left\|p_{0}\right\|_{\infty}=1$. Since $p_{0}$ was minimal we infer $\|p\|_{2}=\left\|p_{0}\right\|_{2}$. This proves the lemma.

LEMMA 1.4. $\operatorname{dim} W_{n}\left(S_{d}\right)=\alpha(d, 2 n)^{-1}$.

Proof. It follows from Proposition 1.1 that $\operatorname{dim} W_{n}\left(S_{d}\right)=\operatorname{trace} P_{n}$. Since $P_{n}$ is a projection its trace equals the square of its Hilbert-Schmidt norm, which, as is well known, equals

$$
\alpha(d, 2 n)^{-2} \int_{S_{d}} \int_{S_{d}}|\langle\zeta, \eta\rangle|^{2 n} d \sigma(\zeta) d \sigma(\eta)=\alpha(d, 2 n)^{-1} .
$$

Now we are ready to prove the theorem.

Proof of TheOREM 1.2. We apply (1) for $X=W_{n}^{2}\left(S_{d}\right)$ and $Y=W_{n}^{\infty}\left(S_{d}\right)$. Using (2), Proposition 1.1 and Lemma 1.4 we obtain

$$
\begin{aligned}
d\left(W_{n}^{2}\left(S_{d}\right), W_{n}^{\infty}\left(S_{d}\right)\right) & \geqslant \lambda\left(W_{n}^{2}\left(S_{d}\right)\right) \lambda\left(W_{n}^{\infty}\left(S_{d}\right)\right)^{-1} \\
& \geqslant 2^{1-d}(\sqrt{\pi} / 2) \sqrt{\alpha(d, 2 n)^{-1}} .
\end{aligned}
$$

Let $I$ denote the identity map from $W_{n}^{\infty}\left(S_{d}\right)$ into $W_{n}^{2}\left(S_{d}\right)$. By the definition of the Banach-Mazur distance we have $\|I\| \cdot\left\|I^{-1}\right\| \geqslant d\left(W_{n}^{2}\left(S_{d}\right), W_{n}^{\infty}\left(S_{d}\right)\right)$. Lemma 1.3 gives $\left\|I^{-1}\right\|=\sqrt{\alpha(d, 2 n)^{-1}}$ so by (3) we have

$$
\|I\| \geqslant 2^{1-d} \sqrt{\pi} / 2
$$

This inequality proves the theorem.

REMARK. Actually $d\left(W_{n}^{2}\left(S_{d}\right), W_{n}^{\infty}\left(S_{d}\right)\right)=\|I\| \cdot\left\|I^{-1}\right\|$. This follows from [1, Lemma 4.6].

COROLlaRY 1.5. id: $H_{\infty}\left(B_{d}\right) \rightarrow H_{1}\left(B_{d}\right)$ is not a compact operator.

Proof. Polynomials $p_{n}$ exhibited in Theorem 1.2 are orthogonal. Moreover by the Schwartz inequality, $\sqrt{\pi} 2^{-d} \leqslant\left\|p_{n}\right\|_{2} \leqslant \sqrt{\left\|p_{n}\right\|_{1} \cdot\left\|p_{n}\right\|_{\infty}}$, so $\left\|p_{n}\right\|_{1} \geqslant \pi 4^{-d}$. This implies that id: $H_{\infty}\left(B_{d}\right) \rightarrow H_{1}\left(B_{d}\right)$ is not a compact operator. 
Proposition 1.6. Let $\left(n_{k}\right)$ be a lacunary sequence of natural numbers and let $p_{n_{k}} \in W_{n_{k}}\left(S_{d}\right)$ be such that $\left\|p_{n_{k}}\right\|_{\infty}=1$ and $\left\|p_{n_{k}}\right\|_{1} \geqslant c, k=0,1,2 \ldots$. Then there exist constants $A$ and $B$ such that

$$
A^{-1}\left(\sum_{k}\left|a_{k}\right|^{2}\right)^{1 / 2} \leqslant\left\|\sum_{k} a_{k} p_{n_{k}}\right\|_{1} \leqslant A\left(\sum_{k}\left|a_{k}\right|^{2}\right)^{1 / 2}
$$

and for $f \in H_{1}\left(B_{d}\right)$,

$$
\left\|\sum_{k} \frac{\left\langle f, p_{n_{k}}\right\rangle}{\left\|p_{n_{k}}\right\|_{2}^{2}} p_{n_{k}}\right\|_{1} \leqslant B\|f\|_{1} .
$$

Proof. Let $\mathscr{P}_{d}$ be a complex projective space, i.e. $\mathscr{P}_{d}=S_{d} / T(T$ denotes the unit circle). The space $\mathscr{P}_{d}$ has a natural invariant probability measure $\mu$. Moreover for $f \in L_{1}(\sigma)$ we have

$$
\int_{S_{d}} f(\zeta) d \sigma(\zeta)=\int_{\mathscr{P}_{d}} \int_{T} f(t \cdot \xi) d t d \mu(\xi) .
$$

Using (6) and the Khintchine inequality we obtain

$$
\int_{S_{d}}\left|\sum_{k} a_{k} p_{n_{k}}(\zeta)\right| d \sigma(\zeta) \sim \int_{\mathscr{P}_{d}} \sqrt{\sum\left|a_{k}\right|^{2}\left|p_{n_{k}}(\xi)\right|^{2}} d \mu(\xi) .
$$

Since $\left\|p_{n_{k}}\right\|_{\infty}=1,(7)$ does not exceed $\sqrt{\sum\left|a_{k}\right|^{2}}$. On the other hand,

$$
\begin{aligned}
c \sum_{k}\left|a_{k}\right|^{2} & \leqslant \int_{\mathscr{P}_{d}} \sum_{k}\left|a_{k}\right|^{2}\left|p_{n_{k}}(\xi)\right|^{2} d \mu(\xi) \\
& \leqslant \sup _{\xi \in \mathscr{P}_{d}} \sqrt{\sum_{k}\left|a_{k}\right|^{2}\left|p_{n_{k}}(\xi)\right|^{2}} \cdot \int_{\mathscr{P}_{d}} \sqrt{\sum_{k}\left|a_{k}\right|^{2}\left|p_{n_{k}}(\xi)\right|^{2}} d \mu(\xi) \\
& \leqslant \sqrt{\Sigma_{k}\left|a_{k}\right|^{2}} \int_{\mathscr{P}_{d}} \sqrt{\Sigma_{k}\left|a_{k}\right|^{2}\left|p_{n_{k}}(\xi)\right|^{2}} d \mu(\xi) .
\end{aligned}
$$

This implies

$$
c \sqrt{\Sigma_{k}\left|a_{k}\right|^{2}} \leqslant \int_{\mathscr{P}_{d}} \sqrt{\Sigma_{k}\left|a_{k}\right|^{2}\left|p_{n_{k}}(\xi)\right|^{2}} d \mu(\xi) .
$$

So by (7) we get (4).

To prove (5) we state the obvious

LEMMA 1.7. Let $\Lambda=\left(\lambda_{n}\right)$ be a continuous multiplier from $H_{1}(D)$ into itself. Then for $f \in H_{1}\left(B_{d}\right), f=\sum_{k=0}^{\infty} f_{k}, f_{k} \in W_{k}\left(S_{d}\right)$, the operator $\check{\Lambda}$ defined as $\check{\Lambda}(f)=\sum_{k=0}^{\infty} \lambda_{k} f_{k}$ is a continuous operator from $H_{1}\left(B_{d}\right)$ into itself.

The proof follows immediately from (6). Using Lemma 1.7 and the classical one-dimensional Paley theorem (cf. [3]) we obtain that the operator $Q\left(\Sigma_{k} f_{k}\right)=\Sigma_{k} f_{n_{k}}$ is a continuous projection in $H_{1}\left(B_{d}\right)$. Moreover as in (7) we obtain

$$
\left\|\sum_{k} f_{n_{k}}\right\|_{1} \sim \int_{S_{d}}\left(\sum_{k}\left|f_{n_{k}}\right|^{2}\right)^{1 / 2} \text {. }
$$


Hence

$$
\begin{aligned}
\left\|\sum_{k} \frac{\left\langle f, p_{n_{k}}\right\rangle}{\left\|p_{n_{k}}\right\|_{2}^{2}} p_{n_{k}}\right\|_{1} & =\left\|\sum_{k} \frac{\left\langle f_{n_{k}}, p_{n_{k}}\right\rangle}{\left\|p_{n_{k}}\right\|_{2}^{2}} p_{n_{k}}\right\|_{1} \leqslant A\left(\sum_{k} \frac{\left|\left\langle f_{n_{k}}, p_{n_{k}}\right\rangle\right|^{2}}{\left\|p_{n_{k}}\right\|_{2}^{4}}\right)^{1 / 2} \\
& \leqslant \frac{A}{c}\left(\sum_{k}\left\|f_{n_{k}}\right\|_{1}^{2}\right)^{1 / 2} \leqslant \frac{A}{c} \int\left(\sum_{k}\left|f_{n_{k}}\right|^{2}\right)^{1 / 2} \leqslant B\|f\|_{1} .
\end{aligned}
$$

Since Theorem 1.2 asserts that we can satisfy the assumptions of Proposition 1.6 we have

COROllaRy 1.8. The space $H_{1}\left(B_{d}\right)$ contains a complemented copy of a Hilbert space.

REMARK. We do not know the isomorphic type of a Banach space $\operatorname{Im} Q$.

Theorem 1.2 also gives some information about the space $\Re\left(B_{d}\right)$ of all Bloch functions on $B_{d}$. Definitions and basic properties of the space $\mathscr{B}\left(B_{d}\right)$ are contained in [7]. From Theorems 1.5 and 4.10 of [7] it follows that $\Sigma_{k} a_{k} p_{2^{k}} \in \mathscr{B}\left(B_{d}\right)$ for every $\left(a_{k}\right) \in l_{\infty}$. On the other hand Proposition 1.5 shows that $\Sigma_{k} a_{k} p_{2^{k}} \in H_{p}\left(B_{d}\right)$, $0<p<\infty$, if and only if $\left(a_{k}\right) \in l_{2}$. These two facts yield

Corollary 1.9. The space $\left.\oiint_{(}\right)$is not contained in $H_{p}\left(B_{d}\right), 0<p<\infty$.

This corollary answers the question from [7, p. 250].

We conclude this section with the following

REMARK 1.10. In $\$ 7.2$ of [5] W. Rudin uses certain $n$-homogeneous polynomials $F_{n}(z)$ (cf. Proposition 7.2.8 of [5]) in order to construct examples that illustrate possible boundary behavior of analytic functions in $B_{2}$ and $B_{3}$. He remarks that "similar examples undoubtedly exist when $d>3$ but different constructions would be required for them." These "different constructions" are provided by Theorem 1.2. When one applies arguments from [5,7.2.9,7.2.10 and 7.2.11] to polynomials $p_{n}$ instead of polynomials $F_{n}$, one obtains the same (or even stronger) results for the ball of arbitrary dimension.

2. In this section we consider several natural operators between $W_{n}^{\infty}\left(S_{2}\right)$ and $l_{\infty}^{n+1}$, and show that those operators do not give good estimates for $d\left(W_{n}^{\infty}\left(S_{2}\right), l_{\infty}^{n+1}\right)$. Our results, although nonconclusive from the Banach space point of view, give some new information about interpolating polynomials in $W_{n}\left(S_{2}\right)$.

We will call a set $A=\left\{\eta_{0}, \eta_{1}, \ldots, \eta_{n}\right\} \subset S_{2}$ admissible if $\lambda \eta_{i}=\eta_{j}$ if and only if $i=j$ and $\lambda=1$. It is clear that for every sequence of complex numbers $\left(a_{0}, a_{1}, \ldots, a_{n}\right)$ there exists a uniquely determined polynomial $p \in W_{n}\left(S_{2}\right)$ such that $p\left(\eta_{j}\right)=a_{j}$, $j=0,1, \ldots, n$. In particular for every admissible set $A$ we have a set of polynomials $v_{j} \in W_{n}\left(S_{2}\right), j=0,1, \ldots, n$, such that $v_{j}\left(\eta_{i}\right)=\delta_{j i}\left(\delta_{j i}\right.$ being the Kronecker symbol). If $\eta_{j}=\left(p_{j}, q_{j}\right)$ then polynomials $v_{r}$ are given as

$$
v_{r}(z, w)=\frac{\prod_{j \neq r} q_{j} z-p_{j} w}{\prod_{j \neq r} q_{j} p_{r}-p_{j} q_{r}} .
$$


We start with the following easy

THEOREM 2.1. For an arbitrary admissible set $A$ there exists a point $\zeta_{0} \in S_{2}$ such that

$$
\sum_{r=0}^{n}\left|v_{r}\left(\zeta_{0}\right)\right| \geqslant \frac{1}{2} \sqrt{n+1}
$$

Proof. By suitable renumeration and rotation we can assume that

$$
\prod_{j=1}^{n}\left|q_{j} p_{0}-p_{j} q_{0}\right|=\max _{r} \prod_{j \neq r}\left|q_{j} p_{r}-p_{j} q_{r}\right|
$$

and that $\eta_{0}=1$. Then

$$
\begin{aligned}
\sum_{r=0}^{n}\left|v_{k}(z, w)\right| & =\left|v_{0}(z, w)\right|+\sum_{r=1}^{n}\left|\frac{q_{0} z-p_{0} w}{q_{r} z-p_{r} w}\right| \frac{\prod_{j=1}\left|q_{j} z-p_{j} w\right|}{\prod_{j \neq r}\left|q_{j} p_{r}-p_{j} q_{r}\right|} \\
& \geqslant\left|v_{0}(z, w)\right|\left(1+\sum_{r=1}^{n}\left|\frac{q_{0} z-p_{0} w}{q_{r} z-p_{r} w}\right|\right) \\
& \geqslant\left|v_{0}(z, w)\right|(1+n|w|) \geqslant(n+1)|w|\left|v_{0}(z, w)\right| .
\end{aligned}
$$

Since $\frac{1}{2 \pi} \int_{0}^{2 \pi} v_{o}\left(z, e^{i \theta} w\right) d \theta=z^{n}$ we infer that there exists a point $\zeta_{0}=(\sqrt{1-1 / n}$, $\left.e^{i \varphi} / \sqrt{n}\right)$ such that $\left|v_{0}\left(\zeta_{0}\right)\right| \geqslant\left(1-\frac{1}{n}\right)^{n / 2}$. For this point we have

$$
\sum_{r=0}^{n}\left|v_{r}\left(\zeta_{0}\right)\right| \geqslant \frac{1}{\sqrt{n}}(n+1)\left(1-\frac{1}{n}\right)^{n / 2} \geqslant \frac{1}{2} \sqrt{n+1} .
$$

Let us introduce some geometric notions on $S_{2}$. We will use the rotation-invariant pseudometric $\rho(\zeta, \eta)=\sqrt{1-|\langle\zeta, \eta\rangle|^{2}}$. In fact it is a natural metric on two-dimensional complex projective space. We will use the parametrisation of $S_{2}$ as $z=\sqrt{\rho} e^{i \psi}$, $w=\sqrt{1-\rho} e^{i \theta}, 0 \leqslant \rho \leqslant 1,0 \leqslant \psi \leqslant 2 \pi, 0 \leqslant \theta \leqslant 2 \pi$. In this parametrisation $d \sigma=$ $\left(1 / 4 \pi^{2}\right) d \rho d \psi d \theta$. Using this parametrisation one sees that for small $r, \sigma(B(\eta, r)) \sim$ $r^{2}$. By $P\left(\eta, r_{1}, r_{2}\right)$ we will denote the set $\left\{\zeta \in S_{2}: r_{1} \leqslant \rho(\zeta, \eta) \leqslant r_{2}\right\}$. Now we are ready to consider the interpolating polynomials $v_{r}(z, w)$ in $W_{n}^{1}\left(S_{2}\right)$. We have the following

Proposition 2.2. There exists a constant $c>0$ such that for every admissible set $A \subset S_{2}$ there exists a $k, k=0,1, \ldots, n$, such that

$$
\int_{S_{2}}\left|v_{k}(z, w)\right| d \sigma(z, w) \geqslant \frac{c}{\sqrt{n}} .
$$

The proof of the following elementary lemma can be found in [2, Formula 2620].

LEMMA 2.3 .

$$
\int_{0}^{2 \pi} \ln \left|1+\alpha e^{i \theta}\right| d \theta= \begin{cases}0 & \text { if } 0 \leqslant \alpha \leqslant 1, \\ 2 \pi \ln \alpha & \text { if } 1 \leqslant \alpha .\end{cases}
$$

Using this lemma we obtain

LEMMA 2.4. For given $\eta=\left(a_{1}, a_{2}\right)$ and $\zeta=\left(b_{1}, b_{2}\right)$ in $S_{2}$ let

$$
\varphi(z, w)=\left(z b_{2}-w b_{1}\right) /\left(a_{1} b_{2}-a_{2} b_{1}\right) \text {. }
$$


Then

$$
\int_{P(\eta, \sqrt{2 / n}, \sqrt{4 / n})} \ln |\varphi(z, w)| d \sigma(z, w) \geqslant-\frac{6}{n^{2}} .
$$

If $\rho(\eta, \zeta) \leqslant 1 / \sqrt{n}$ then

$$
\int_{P(\eta, \sqrt{2 / n}, \sqrt{4 / n})} \ln |\varphi(z, w)| d \sigma(z, w) \geqslant \frac{d}{n}
$$

for some constant $d>0$ independent of the set $A$.

Proof. For simplicity let us assume $n \geqslant 8$ and $\eta=1$. Let us denote $\alpha=\left|b_{1} / b_{2}\right|$.

$$
\begin{aligned}
& \int_{P(1, \sqrt{2 / n}, \sqrt{4 / n})} \ln |\varphi(z, w)| d \sigma(z, w) \\
& \quad=\frac{1}{2 \pi} \int_{1-4 / n}^{1-2 / n} \int_{0}^{2 \pi} \ln \left|\rho^{1 / 2}+\alpha \sqrt{1-\rho} e^{i \psi}\right| d \rho d \psi \\
& \quad=\frac{1}{2} \int_{1-4 / n}^{1-2 / n} \ln \rho d \rho+\frac{1}{2 \pi} \int_{1-4 / n}^{1-2 / n} \int_{0}^{2 \pi} \ln \left|1+\alpha\left(\frac{1-\rho}{\rho}\right)^{1 / 2} e^{i \psi}\right| d \psi d \rho
\end{aligned}
$$

Using Lemma 2.3 we see that (8) is greater than $\frac{1}{2} \int_{1-4 / n}^{1-2 / n} \ln \rho d \rho \geqslant-6 / n^{2}$.

If $\rho(1, \zeta) \leqslant 1 / \sqrt{n}$ then $\alpha \geqslant \sqrt{n-1}$ so (8) is greater than

$$
\begin{aligned}
\frac{1}{2} \int_{1-4 / n}^{1-2 / n} \ln \rho d \rho & +\frac{1}{2 \pi} \int_{1-4 / n}^{1-2 / n} \int_{0}^{2 \pi} \ln \left|1+\sqrt{n-1}\left(\frac{1-\rho}{\rho}\right)^{1 / 2} e^{i \psi}\right| d \psi d \rho \\
& =\frac{1}{2} \int_{1-4 / n}^{1-2 / n} \ln \rho d \rho+\int_{1-4 / n}^{1-2 / n} \ln \sqrt{n-1}\left(\frac{1-\rho}{\rho}\right)^{1 / 2} d \rho \geqslant \frac{d}{n} .
\end{aligned}
$$

Proof of Proposition 2.2. We define $k$ by the condition

$$
\prod_{j \neq k}^{n}\left|p_{j} q_{k}-q_{j} p_{k}\right|=\min _{r} \prod_{j \neq r}\left|p_{j} q_{r}-q_{j} p_{r}\right|
$$

We may assume $k=0$. We may also find a set $M \subset\{0,1, \ldots, n\}$ such that for $j \in M, B\left(\eta_{j}, 2 / \sqrt{n}\right)$ are disjoint and $\cup_{j \in M} B\left(\eta_{j}, 2 / \sqrt{n}\right)$ contains at least $(n+1) / 100$ points from $A$.

Let $P_{j}=P\left(\eta_{j}, \sqrt{2 / n}, \sqrt{4 / n}\right), j \in M$. For $(z, w) \in P_{j}$ we have

$$
\left|v_{0}(z, w)\right| \geqslant\left|\frac{p_{j} w-q_{j} z}{p_{0} w-q_{0} z}\right|\left|v_{j}(z, w)\right| \geqslant \frac{1}{\sqrt{n}}\left|v_{j}(z, w)\right| .
$$

Using Lemma 2.4 we see that

$$
\begin{aligned}
\int_{P_{j}} \ln \left|v_{j}(z, w)\right| d \sigma(z, w) & =\sum_{k \neq j} \int_{P_{j}} \ln \left|\frac{q_{k} z-p_{k} w}{q_{k} p_{j}-p_{j} q_{k}}\right| \\
& \geqslant-6\left(n+1-c_{j}\right) / n^{2}+c_{j} d / n
\end{aligned}
$$

where $c_{j}$ denotes the number of points in $A \cap B\left(\eta_{j}, 1 / \sqrt{n}\right)$. Since

$$
\ln \left(\frac{1}{\sigma\left(P_{j}\right)} \int_{P_{j}}\left|v_{j}(z, w)\right| d \sigma(z, w)\right) \geqslant \frac{1}{\sigma\left(P_{j}\right)} \int_{P_{j}} \ln \left|v_{j}(z, w)\right| d \sigma(z, w)
$$


we infer that

$$
\begin{aligned}
\int_{P_{j}}\left|v_{j}\right| & \geqslant \sigma\left(P_{j}\right) \exp \left(\sigma\left(P_{j}\right)^{-1}\left(\frac{c_{j} d}{n}-\frac{6\left(n+1-c_{j}\right)}{n^{2}}\right)\right) \\
& \geqslant \frac{2}{n} \exp \left(2^{-1} c_{j} d-\frac{3\left(n+1-c_{j}\right)}{n}\right) \geqslant \frac{b}{n} \exp 2^{-1} c_{j} d \geqslant \frac{b c_{j}}{n} .
\end{aligned}
$$

Finally we obtain

$$
\begin{aligned}
\int_{S_{2}}\left|v_{0}(z, w)\right| d \sigma(z, w) & \geqslant \sum_{j \in M} \int_{P_{j}}\left|v_{0}(z, w)\right| d \sigma(z, w) \\
& \geqslant \sum_{j \in M} \frac{1}{\sqrt{n}} \int_{P_{j}}\left|v_{j}(z, w)\right| d \sigma(z, w) \geqslant \sum_{j \in M} \frac{1}{\sqrt{n}} \frac{b \cdot c_{j}}{n} \geqslant c \frac{1}{\sqrt{n}} .
\end{aligned}
$$

This completes the proof of the proposition.

THEOREM 2.5. There exists a constant $c>0$ such that for every admissible set $A=\left\{\eta_{0}, \eta_{1}, \ldots, \eta_{n}\right\}$ there exists a sequence of numbers $\varepsilon_{0}, \varepsilon_{1}, \ldots, \varepsilon_{n}, \varepsilon_{i}= \pm 1$, such that

$$
\sup _{\zeta \in S_{2}}\left|\sum_{i=0}^{n} \varepsilon_{i}\left\langle\zeta, \eta_{i}\right\rangle^{n}\right| \leqslant \frac{c}{\sqrt{n}}
$$

Proof. Using the projection exhibited in Proposition 1.1 we can identify $W_{n}^{\infty}\left(S_{2}\right)^{*}$ with $W_{n}^{1}\left(S_{2}\right)$ with the natural duality. Let $T: l_{\infty}^{n+1} \rightarrow W_{n}^{\infty}\left(S_{2}\right)$ be defined by $T\left(e_{j}\right)=\left\langle\cdot, \eta_{j}\right\rangle^{n}$. We have to prove that $\left\|T^{-1}\right\| \geqslant \sqrt{n} / c$. But $\left\|T^{-1}\right\|=\left\|\left(T^{*}\right)^{-1}\right\|$. Since

$$
\int_{S_{2}} v_{j}(\xi)\left\langle\zeta, \eta_{k}\right\rangle^{n} d \sigma(\zeta)= \begin{cases}(n+1)^{-1} & \text { if } j=k \\ 0 & \text { if } j \neq k\end{cases}
$$

we see that $\left(T^{*}\right)^{-1}: l_{1}^{n+1} \rightarrow W_{n}^{1}\left(S_{2}\right)$ is defined by $\left(T^{*}\right)^{-1}\left(e_{j}\right)=(n+1) v_{j}$. Proposition 2.2 implies that $\left\|\left(T^{*}\right)^{-1}\right\| \geqslant c \cdot \sqrt{n}$. This gives the proof of the theorem.

REMARK. Theorem 2.5 means that for every admissible set $A$ the operator $T$ : $l_{\infty}^{n+1} \rightarrow W_{n}^{\infty}\left(S_{2}\right)$ defined by $T\left(e_{j}\right)=\left\langle\cdot, \eta_{j}\right\rangle^{n}$ satisfies $\|T\| \cdot\left\|T^{-1}\right\| \geqslant c \sqrt{n}$. On the other hand Theorem 2.1 gives that an operator $S: W_{n}^{\infty}\left(S_{2}\right) \rightarrow l_{\infty}^{n+1}$ given by $S(f)=\left(f\left(\eta_{j}\right)\right)_{j=0}^{n}$ satisfies $\|S\| \cdot\left\|S^{-1}\right\| \geqslant c \sqrt{n}$. So those two natural operators give very bad estimates for $d\left(W_{n}^{\infty}\left(S_{2}\right), l_{\infty}^{n+1}\right)$.

In our next proposition we examine one more operator.

Proposition 2.6. Let $V: l_{\infty}^{n+1} \rightarrow W_{n}^{\infty}\left(S_{2}\right)$ be given by

$$
V\left(e_{j}\right)(z, w)=z^{j} w^{n-j} /\left\|z^{j} w^{n-j}\right\|_{\infty} .
$$

Then $\|V\| \geqslant c \sqrt{n}$. 
Proof. It is easily seen that $\left\|z^{j} w^{n-j}\right\|_{\infty}=((n-j) / n)^{(n-j) / 2}(j / n)^{j / 2}$. So we have

$$
\begin{aligned}
\|V\| & =\sup _{(z, w) \in S_{2}} \sum_{j=0}^{n} \frac{|z|^{j}|w|^{n-j}}{((n-j) / n)^{(n-j) / 2}(j / n)^{j / 2}} \\
& \geqslant \sum_{j=0}^{n} \frac{(1 / \sqrt{2})^{n}}{((n-j) / n)^{(n-j) / 2}(j / n)^{j / 2}} \\
& \geqslant \sum_{|j-n / 2| \leqslant \sqrt{n}} \frac{(n / 2)^{n / 2}}{(n-j)^{(n-j) / 2}(j)^{j / 2}} \geqslant 0.06 \sqrt{n} .
\end{aligned}
$$

To conclude our paper we will give a sketch of a direct proof of Theorem 1.2.

LEMMA 2.7. Let $A=\left\{\eta_{0}, \eta_{1}, \ldots, \eta_{n}\right\}$ be an admissible set such that

$$
\sup _{i \neq j}\left|\left\langle\eta_{j}, \eta_{i}\right\rangle\right|<\sqrt{1-c / n} .
$$

Then

$$
\sup _{\zeta \in S_{2}} \sum_{j}\left|\left\langle\zeta, \eta_{j}\right\rangle^{n}\right| \leqslant d(c) \text {. }
$$

Proof. Our condition implies that for every $\zeta \in S_{2}$ the ball $B(\zeta, \sqrt{k / n})$ contains at most $b \cdot k$ points from $A$. It implies that

$$
\sum_{i}\left|\left\langle\zeta, \eta_{i}\right\rangle^{n}\right| \leqslant \sum_{k} b \cdot k \cdot\left(1-\frac{k}{n}\right)^{n} \leqslant d(c) .
$$

Easy consideration involving measures of $B(1, \rho)$ shows that, in fact, for some constant $c$ one can find for arbitrary $n$ an admissible set satisfying (9). For this set we have $\left(r_{k}(t)\right.$ are Rademacher functions)

$$
\int_{0}^{1}\left\|\sum_{k=0}^{n} r_{k}(t)\left\langle\zeta, \eta_{k}\right\rangle^{n}\right\|_{2}^{2}=(n+1)\left\|\langle\zeta, 1\rangle^{n}\right\|_{2}^{2} \geqslant c .
$$

It implies that for some choice of signs $\left(\varepsilon_{n}\right)$ we have

$$
\left\|\sum_{k=0}^{n} \varepsilon_{k}\left\langle\zeta, \eta_{k}\right\rangle^{n}\right\|_{2} \geqslant c_{1}
$$

and by Lemma 2.7 we also have

$$
\left\|\sum_{k=0}^{n} \varepsilon_{k}\left\langle\zeta, \eta_{k}\right\rangle^{n}\right\|_{\infty} \leqslant c_{2}
$$

REMARK. Clearly the above proof can be adopted also for $d>2$.

REMARK. The space $W_{n}^{\infty}\left(S_{2}\right)$ can be identified with certain weighted space of polynomials in one variable. Namely we put $z / w=v$ and we obtain

$$
\left|\sum_{k=0}^{n} a_{k} z^{k} w^{n-k}\right|=\frac{1}{\left(1+|v|^{2}\right)^{n / 2}}\left|\sum_{k=0}^{n} a_{k} v^{b}\right| .
$$

This approach was not very illuminating for us. 


\section{REFERENCES}

1. Y. Benyamini and Y. Gordon, Random factorisation of operators between Banach spaces, J. Analyse Math. 39(1981), 45-74.

2. G. S. Carr, Formulas and theorems in pure mathematics, Chelsea, New York, 1970.

3. P. Duren, Theory of $H_{p}$ spaces, Academic Press, New York, 1970.

4. A. Pietsch, Operator ideals, VEB Deutscher Verlag, Berlin, 1978.

5. W. Rudin, Function theory in the unit ball of $\mathbf{C}^{n}$, Springer-Verlag, Berlin and New York, 1980.

6. D. Rutowitz, Some parameters associated with finite dimensional Banach spaces, J. London Math. Soc. 40 (1965), 241-255.

7. R. M. Timoney, Bloch functions in several complex variables. I, Bull. London Math. Soc. 12 (1980), 241-267.

8. A. Zygmund, Trigonometric series, Cambridge Univ. Press., New York, 1979.

Department of Mathematics, Warsaw University, Warsaw, Poland (Current address of J. Ryll)

Department of Mathematics, The University of TeXas, Austin, TeXas 78712

Current address (P. Wojtaszczyk): Institute of Mathematics, Polish Academy of Sciences, 00-950 Warsaw, Poland 